\title{
AN ECONOMIC MODEL OF SCHOOL-BASED BEHAVIORAL INTERVENTIONS TO PREVENT SEXUALLY TRANSMITTED INFECTIONS
}

Keith Cooper

e-mail:kc@soton.ac.uk
Jonathan Shepherd, Jo Picot, Jeremy Jones, Josephine Kavanagh, Angela Harden, Elaine Barnett-Page, Andrew Clegg, Debbie Hartwell, Geoff Frampton, Alison Price

Objectives: Reducing sexually transmitted infections (STI) and teenage pregnancy through effective health education is a high priority for health policy. Behavioral interventions which teach skills to practice safer sex may reduce the incidence of STls. We evaluated the cost-effectiveness of school-based behavioral interventions in young people.

Methods: We developed an economic model to estimate the total number of STI cases averted, consequent gain in health related quality of life (HRQoL) and savings in medical costs, based on changes in sexual behavior. The parameters for the model were derived from a systematic literature search on the intervention effectiveness, epidemiology of STls, sexual behavior and lifestyles, HRQoL and health service costs.

Results: The costs of providing teacher-led and peer-led behavioral interventions were $€ 5.16$ and $€ 18$ per pupil, respectively. For a cohort of 1000 boys and 1000 girls aged 15 years, the model estimated that the behavioral interventions would avert two STI cases and save 0.35 Quality Adjusted Life Years (QALYS). Compared to standard education, the incremental cost-effectiveness of the teacher-led and peer-led interventions was €24,268 and €96,938 per QALY gained, respectively.

Conclusions: School-based behavioral interventions which provide information and teach young people sexual health skills can bring about improvements in knowledge and increased self-efficacy, though these may be limited in terms of impact on sexual behavior. There was uncertainty around the results due to the limited effect of the intervention on behavioral outcomes and paucity of data for other input parameters.

Keywords: cost effectiveness, model, school, sexually transmitted infection

Rates of sexually transmitted infections (STIs) are continuing to rise in the many countries, particularly among young people (10). If undiagnosed and untreated, STIs can lead to serious long-term complications, such as infertility and pelvic inflammatory disease (PID), and, in the case of human immunodeficiency virus (HIV), mortality. The rise in STIs is in part due to greater uptake of testing, which is identifying more infections, but also continuing unsafe sexual behavior in young people $(10 ; 13)$. Often these young people lack the skills and confidence to negotiate safer sex.

The impact of increases in the incidence and prevalence of STIs over recent years has placed great demand on health services and the prevention of STIs and teenage pregnancy is currently a high priority for health policy. To reduce STIs, effective health education strategies need to be developed and implemented to provide knowledge for and change young peoples' sexual behavior.

Behavioral interventions to prevent STIs are often provided in schools and colleges as part of the curriculum, for example in the United Kingdom, these are provided during Sex and Relationship Education (SRE) lessons. There is variability in the topics covered, the format of the lessons, and the staff used to deliver them. Some schools primarily provide factual information, whereas others supplement this with interactive learning, such as role play, condom use demonstrations, and educational theater, sometimes with input from outside agencies, for exam- ple health promotion services. The objective of SRE is to help and support young people through their physical, emotional, and moral development and to enable them to make informed decisions about their lives. However, there is uncertainty and sometimes controversy about how and when sex education should be provided.

Interventions to prevent STIs in young people should be based upon sound evidence. In addition, the costs of such interventions and the gains to health, in terms of the infections averted, associated gains in health-related quality of life (HRQoL), and lives saved, should be assessed. The costeffectiveness of an intervention provides a measure of its value for money, and is part of the evidence used by decision makers. There is a dearth of evidence for the cost-effectiveness of some types of health promotion, particularly social interventions in schools, although decision makers need such evidence to inform the allocation of scarce resources. The objective of this study is to assess the cost-effectiveness of school-based behavioral interventions for the prevention of STIs in young people through the development of an economic model.

\section{METHODS}

\section{Cost-Effectiveness Analysis}

The cost-effectiveness of two types of school-based behavioral interventions were assessed: teacher-led and peer-led. Both 
interventions provide factual information about STIs in addition to the teaching of skills associated with the practice of safer sex. These two interventions were considered to be broadly representative of studies of the effectiveness of behavioral interventions to prevent STIs that were included in our systematic review (22). The teacher-led intervention comprised twenty sessions taking place over a 2-year period (10 sessions at age 13-14 years, and 10 sessions at age 14-15 years). The intervention was intended to reduce unsafe sex and unwanted pregnancies and improve the quality of sexual relationships. It involved active learning (small group work and games), information leaflets on sexual health, and development of skills, primarily through the use of interactive video and role playing. The peer-led intervention comprised three sessions led by peer educators lasting 1 hour each, over one school term. The sessions covered relationships, sexually transmitted infections, and use of condoms and contraception. They were designed to be informal using small group work, role plays, and condom use skills demonstrations.

The comparator for both of these interventions was standard sexual health education, which is generally provided by teachers in British schools as part of the SRE curriculum. Standard sexual health education generally provides basic information on STIs and sexual health, but does not necessarily teach safer sex negotiation skills. It is, therefore, the teaching of safer sex skills and other broader activities that distinguishes the behavioral intervention from standard education.

The model was constructed in Microsoft Excel according to standard methods (18). Only direct National Health Service (NHS) and Personal Social Services (PSS) costs were included; hence, the model was from the perspective of the UK NHS and PSS. In the base case analysis, a cohort of young people aged 15 years old receive the teacher-led intervention. The model estimates the costs and benefits from averted STI cases for the intervention and, hence, the cost-effectiveness of the intervention compared with standard sexual health education.

We adapted a previously developed Bernoulli statistical model (28) as it estimates the effect of changes in sexual behavior in terms of STIs averted. The Bernoulli model of HIV transmission is a cumulative probability equation that describes the probability of HIV infection based upon HIV prevalence $(\pi)$, single act transmission probability $(\alpha)$, condom effectiveness (e) and condom use (f), number of sexual episodes (n), and number of sexual partners $(\mathrm{m})$. For example, the estimated probability of an uninfected person becoming infected is $\mathrm{P}$,

$$
P=1-\left\{(1-\pi)+\pi|1-\alpha(1-e f)|^{n}\right\}^{m} .
$$

The model estimates the probability of becoming infected for the intervention and comparator groups according to changes in parameters that may be affected by the intervention, that is, condom use, number of sexual partners, number of sexual episodes. The number of cases averted is estimated by multiplying the results by the number of people who receive the intervention. These cases averted would have, in turn, infected further individuals, that is, through secondary transmission. The number of cases averted through secondary transmission is estimated by multiplying the risk of becoming infected by the number of cases averted through primary transmission.

The model estimates the number of cases averted for HIV, chlamydia, gonorrhea, and genital warts, according to the risk of infection and the proportion of sexually active individuals who receive the intervention. For each STI case averted, there is a HRQoL loss and resource use cost associated due to complications, such as PID or infertility. The data and assumptions used to derive the model parameters are described briefly below and in more detail elsewhere (22).

The total number of STI cases averted, consequent qualityadjusted life-year (QALY) gain, cost of the intervention, and the saving in medical costs is estimated for males and females for one year. Thus the cost-effectiveness is calculated,

$$
\text { Cost effectiveness }=\frac{\text { Cost of intervention }- \text { Saving in medical costs }}{\text { QALYs gained }} .
$$

This outcome measure gives a guide to whether the intervention is good value for money for healthcare decision makers. For the UK NHS, an intervention with a cost-effectiveness ratio less than $£ 30,000$ ( $€ 36,000)$ per QALY is generally considered to be cost-effective.

\section{Parameters Used in the Model}

The parameters used in the model were derived from a range of sources providing epidemiological and quality of life data and are described in the following section. To identify data to populate the model, systematic searches were conducted to locate studies on the natural history and epidemiology of STIs, sexual behavior and lifestyles of the target population, HRQoL, and costs. Web sites of relevant organizations were also searched and contact was made with experts in the field to identify data. The baseline clinical data were estimated from administrative databases for the United Kingdom, and prospective studies. The HRQoL data have been taken from previous utility studies using validated tools for groups of patients who developed STI complications. Costs were derived from published studies (where available), and from national and local NHS unit costs. The quality of data used for the model varied. Generally, there were few data for children younger than 18 years old. Where there were no data, assumptions were made from existing data. The parameters used in the model are shown in Table 1.

The prevalence of STIs in young people was taken from the National Chlamydia Screening program $(5 ; 20)$ and data from the Health Protection Agency $(9 ; 10)$. We estimated the unit cost and QALY loss per case of STI, based on the prevalence of complications of STIs.

In the model, the intervention effects last for 1 year, on the basis that the majority of the trials included in our 
Table 1. Data Parameters Used in the Cost-Effectiveness Model

\begin{tabular}{|c|c|c|c|c|c|c|}
\hline & \multicolumn{4}{|c|}{ Prevalence of STls (5) (20) (9) (10) } & \multicolumn{2}{|c|}{ STI complications' cost and HRQoL ${ }^{a}$} \\
\hline & \multicolumn{2}{|c|}{$<16$ years old } & \multicolumn{2}{|c|}{$>16$ years old } & \multirow{2}{*}{$\begin{array}{l}\text { Medical costs, € } \\
(1)(3)(17)\end{array}$} & \multirow{2}{*}{$\begin{array}{l}\text { QALY loss } \\
(23)(12)(4)\end{array}$} \\
\hline & Male & Female & Male & Female & & \\
\hline Chlamydia & $1.5 \%$ & $7.5 \%$ & $10 \%$ & $12.5 \%$ & 904.04 & 0.27 \\
\hline Gonorrhea & $0.03 \%$ & $0.16 \%$ & $1.25 \%$ & $1.3 \%$ & 904.04 & 0.27 \\
\hline Genital warts & $0.1 \%$ & $0.65 \%$ & $0.53 \%$ & $1.4 \%$ & 675.13 & 0.238 \\
\hline HIV & $0.13 \%$ & $0.06 \%$ & $0.26 \%$ & $0.12 \%$ & 490,385 & 8.4 \\
\hline
\end{tabular}

systematic review of effectiveness assessed outcomes up to 1 year. The effectiveness of the behavioral intervention on condom use was derived through a meta-analysis of the studies of behavioral interventions included in our systematic review and produced a pooled risk ratio (RR) of 1.05 (95 percent confidence interval, 0.92 to 1.20 ) in favor of the behavioral intervention (versus standard education) (22). The pooled RR is not statistically significant, so caution is advised when interpreting these results.

The data used to derive STI transmission probabilities were primarily based on case series. Quinn et al. determined the frequency of Chlamydia trachomatis genital infection within sexual partnerships (19). In the study, chlamydia was transmitted between partners in 68 percent of the couples during a 4-year period. The per episode chlamydia transmission probability was estimated by assuming that the infection would be transmitted within 10 sexual episodes per relationship and resulted in a per-episode transmission probability of 0.11 . Using the same method, the per-episode transmission probability was estimated to be 0.07 for gonorrhea (21) and 0.1 for genital warts (11). We derived the transmission probability for HIV from a literature review on the risk of HIV transmission following an exposure from a known HIV-positive individual (8).

Data on HRQoL associated with STI complications were derived from studies for HIV (25), for chlamydia and gonorrhea $(12 ; 23)$, and for genital warts and cervical cancer (4). These data were for the prevalence of the conditions, the duration of the symptoms, and the HRQoL loss associated with each complication.

The cross-national Health Behavior in School-aged Children (HBSC) survey (29) reported the proportion of sexually active young people in England (males 36 percent; females 40 percent), and their condom use at last intercourse (males 69 percent; females 71 percent). The number of sexual partners that young people have has been estimated, as 2.1 per year for male and 2 per year for females, from a multi-purpose survey with approximately 1,200 adults (aged 16 or over) in private households in Great Britain each month (14). The UK National Survey of Sexual Attitudes and Lifestyles (NATSAL) reported that the number of occasions of heterosexual sex in the past 4 weeks for 16- to 24-year-olds among respondents who had one or more heterosexual partners in the year before interview was 6.9 (SD 9.1) (13). Because data on sexual activity in the under 16 age group are lacking we assumed lower sexual activity for the under 16-year-old age group and that it would be a quarter of the rate seen in the 16- to 24-year-old group, that is, 1.7 episodes per month.

As the analysis reflects an NHS perspective, it uses UKspecific resource use and costing data where available. Cost data were obtained from several primary and secondary sources. Unit costs for the complications of STIs are shown in Table 1. Adams et al. evaluated the cost-effectiveness of the National Chlamydia Screening Program in England (1), estimating the cost of complications based on diagnosis and treatment. The cost of HIV treatment was derived from a cost-effectiveness study for adults with HIV in England of highly active antiretroviral therapy (HAART) compared with two nucleoside reverse transcriptase inhibitors (NRTIs) (17). We updated their analysis to reflect changes in mortality, discount rates, and healthcare costs and derived that individuals with HIV would have 8.4 fewer QALYs than an uninfected individual, with a lifetime discounted medical cost of $€ 490,389$. Individuals infected with human papillomavirus types 16 and 18 are at risk of cervical cancer. The first year cost of cervical cancer was estimated to be $€ 12,557$ per patient (31), which includes all treatment costs. The cost for treating genital warts was approximately $€ 266$ per case (15).

The teacher-led intervention was based upon a Scottish study (the SHARE trial) (30), and comprised a 20 session classroom-based program over 2 years $(10$ sessions at 
Table 2. Base Case Results for the Teacher-Led Behavioral Intervention Compared to Standard Sex Education

\begin{tabular}{|c|c|c|c|c|c|c|c|c|c|c|}
\hline & \multicolumn{2}{|c|}{ HIV } & \multicolumn{2}{|c|}{ Chlamydia } & \multicolumn{2}{|c|}{ Gonorrhea } & \multicolumn{2}{|c|}{ Genital warts } & \multirow[b]{2}{*}{ Pregnancy } & \multirow[b]{2}{*}{ Total } \\
\hline & Male & Female & Male & Female & Male & Female & Male & Female & & \\
\hline Cases control & 0.002 & 0.007 & 7.33 & 10.34 & 0.13 & 1.03 & 0.96 & 0.82 & & 20.71 \\
\hline Cases intervention & 0.001 & 0.006 & 6.57 & 9.22 & 0.11 & 0.92 & 0.90 & 0.77 & & 18.5 \\
\hline Cases averted, total & 0.000 & 0.000 & 0.76 & 1.12 & 0.01 & 0.11 & 0.06 & 0.05 & 0.05 & 2.1 \\
\hline QALY gained & 0.00 & 0.00 & 0.00 & 0.30 & 0.00 & 0.03 & 0.00 & 0.01 & 0.00 & 0.35 \\
\hline Total medical costs averted, $€$ & 46 & 221 & 6 & 1013 & 0 & 101 & 16 & 35 & 308 & 1745 \\
\hline
\end{tabular}

QALY, quality-adjusted life-year; HIV, human immunodeficiency virus.

age 13-14 years, and 10 at age 14-15 years). The peer-led classroom intervention was based upon an English trial (the RIPPLE trial) (24) and comprised three sessions during one term. The costs of teacher-led and peer-led sexual education interventions are based upon the resources used in the SHARE (30) and RIPPLE trials (24). Contact was made with both the SHARE and RIPPLE research teams to request data on costs and resources. Limited data were available from the teams, so most of the resources were estimated by systematically extracting data from the study publications. We updated the costs to present day values using the NHS multiplier for Hospital \& Community Health Services (7). This method converts a cost in a base year to the current year by multiplying the base cost by an index that reflects changes in costs between these years. The training costs for standard sex education were assumed to be minimal as the majority of the training would take place in house, for example during In-Service Training (INSET) days.

For the teacher-led intervention, we estimated resources based on the SHARE trial, where the teachers were taught in groups of thirteen during a 5-day training course run by a health promotion practitioner. For the sixty-nine teachers in the thirteen schools who took part in the trial, the total updated cost of the teacher training was $€ 108,193$ and 4,197 pupils received the program. We assumed that all teachers that taught SRE would receive training and would be retrained every 5 years. The yearly cost for training was $€ 5.16$ per pupil who received the program.

For the peer-led intervention, we estimated the resources based on the RIPPLE trial where there was no training for the teachers involved, only for peer educators. The RIPPLE trial included 4,063 pupils, 463 peer educators, and fourteen schools. The training was undertaken in groups of twelve peer educators per training session over a 2-day intensive course led by a health promotion practitioner. Peer educators were assumed to only teach sex education for 1 year, following which a new cohort of educators will be recruited and trained. The total costs for the training in the RIPPLE trial was estimated to be $€ 73,154$ or $€ 18$ per pupil who received the program.
Table 3. Summary Results for the Teacher-Led and Peer-Led Behavioral Interventions Compared to Standard Sex Education

\begin{tabular}{lrr}
\hline & Teacher-led & Peer-led \\
\hline Total cost of intervention, $€$ & 10,320 & 36,000 \\
Total medical costs averted, € & 1,745 & 1745 \\
Net additional cost, $€$ & 8,575 & 34,255 \\
Cost per case averted (all STI), € & 4,058 & 16,210 \\
Incremental cost per QALY gained, $€$ & 24,268 & 96,938 \\
\hline
\end{tabular}

STI, sexually transmitted infection; QALY, quality-adjusted life-year.

\section{RESULTS}

The model was run with the inputs shown in Table 1 for a cohort of 1,000 boys and 1,000 girls aged 15 years old. In the base case analysis, the costs from the teacher-led behavioral intervention (SHARE) were used and the results are shown in Table 2. These indicate that the teacher-led intervention would have a net additional cost of $€ 8,575$ and avert an extra two STI cases with a corresponding quality of life gain of 0.35 QALY compared with standard sex education. This corresponds to an incremental cost-effectiveness ratio (ICER) of $€ 24,268$ per QALY gained. The results indicate that most of the STI cases averted are of chlamydia (because this was the most prevalent STI) and the largest QALY gain would be for females who avoid infection with chlamydia.

Using the same intervention effect estimate for the peer-led behavioral intervention (RIPPLE), results in the same health gains, in terms of cases averted and QALYs gained, but at a higher cost corresponding to an ICER of $€ 96,938$ per QALY gained (Table 3 ). The peer-led behavioral intervention is less cost-effective than the teacher-led intervention compared with standard sex education. This is due to the peer-led behavioral intervention being much expensive than the teacher-led intervention, due to the need for more frequent training of the peer educators. 
Table 4. Sensitivity Analyses for the STI Model (Teacher-Led Behavioral Intervention Compared to Standard Sex Education)

\begin{tabular}{|c|c|c|c|c|c|c|}
\hline \multirow[b]{2}{*}{ Variable } & \multirow[b]{2}{*}{ Base case } & \multicolumn{2}{|c|}{ Inputs } & \multicolumn{3}{|c|}{ ICER, € / QALY } \\
\hline & & Low & High & Low & High & Range, $€$ \\
\hline IE Condom use (RR) & 1.05 & 0.92 & 1.2 & $-23,190$ & 2,364 & N/A \\
\hline Transmission probability & $11^{a}$ & $3^{\mathrm{a}}$ & $20^{a}$ & 85,669 & 16,068 & 69,601 \\
\hline IE Number of sex partners & 1 & 0.95 & 1.05 & 13,387 & 69,976 & 56,588 \\
\hline Tubal infertility disutility & 0.76 & 0.61 & 0.91 & 51,898 & 15,836 & 36,061 \\
\hline IE Sex episodes per partner & 1 & 0.95 & 1.05 & 15,521 & 45,314 & 29,794 \\
\hline BC Number of sexual partners & 2 & 1.5 & 4 & 36,743 & 7,170 & 29,573 \\
\hline STI prevalence & $7.5^{\mathrm{ab}}$ & $5.3^{\mathrm{ab}}$ & $9.8^{\mathrm{ab}}$ & 34,562 & 12,996 & 21,566 \\
\hline Proportion sexually active & 36 & 25 & 45 & 42,180 & 21,232 & 20,948 \\
\hline BC Sex episodes per partner & 10 & 6 & 14 & 38,345 & 18,785 & 19,560 \\
\hline Intervention cost, € & 5.16 & 3.6 & 6.72 & 15,438 & 33,097 & 17,659 \\
\hline QALY loss per STI case & $0.27^{a}$ & $0.19^{a}$ & $0.35^{a}$ & 34,668 & 18,667 & 16,001 \\
\hline
\end{tabular}

a Values shown for chlamydia. Parameters for other STls were altered by the same magnitude.

${ }^{b}$ Values shown for females

BC base case; IE intervention effect; STI, sexually transmitted infection; QALY, quality-adjusted life-year.

\section{Sensitivity Analysis}

The parameters in the economic model were varied in a series of sensitivity analyses of the base case analysis and the results are shown in Table 4. Where possible, the parameters were varied according to the ranges of the confidence intervals of these parameters, otherwise a suitable range was chosen. Parameter values for all the STIs were altered by the same magnitude but for simplicity only the input parameters for chlamydia are shown in Table 4 . The sensitivity results are nonlinear, as seen for the parameter for the number of sex episodes per partner (episodes varied from 6 to 14). In this case, the ICER for 6 and 14 sex episodes per partner is $€ 38,345$ and $€ 18,785$, respectively, compared with the base case of $€ 24,268$.The results were most sensitive to the intervention effect, the transmission probability, and the number of sexual partners. The base case analysis assumes that the intervention effects last for 1 year. It may be that the intervention effects last for more than 1 year. In the case of the intervention effect lasting 2 years, the ICER decreases to $€ 7,170$ per QALY gained.

A further scenario analysis assessed the effect of the intervention in older teenagers (aged 16-19 years). In this group, there are more sexually active individuals (male, 56 percent; females, 66 percent)(14), who have more sexual episodes per month (6.9 percent) (13) than younger teenagers. However, condom use for sexually active individuals was lower (male, 55 percent, female, 47 percent) (14). The results from the model showed that, in this age group, there are more STI cases averted, QALYs gained, and medical costs averted that in the younger age group. The ICER for this age group is $€ 13,994$ per QALY gained.
We also explored the uncertainty around the model results in more detail using probabilistic sensitivity analyses. The model was run for 1,000 iterations with input values sampled from probability distributions. The parameters were varied according to the ranges used in the deterministic sensitivity analysis. In this analysis, the teacher-led intervention had an ICER between $€ 0$ and $€ 36,000$ per QALY for 48 percent of iterations (Figure 1), more than $€ 36,000$ per QALY for 28 percent of iterations. For 24 percent of iterations, the intervention was associated with a QALY loss. The peer-led intervention had a corresponding lower likelihood of being cost-effectiveness, with an ICER between $€ 0$ and $€ 36,000$ per QALY for 16 percent of iterations.

\section{DISCUSSION}

The results of this economic evaluation indicate that with current evidence, behavioral interventions are likely to lead to a small reduction in risky sexual behavior, which has a corresponding small health benefit, due to avoiding STIs. These interventions are relatively low cost; therefore, if these benefits are sustained they are likely to be good value for money, according to NICE criteria, whereby the cost-effectiveness is in the range $€ 24,000-€ 36,000$ (or lower) per QALY gained (18).

However, there is large uncertainty around the parameter inputs, and hence also around the model results, which make it difficult to draw firm conclusions.

Both the SHARE and RIPPLE studies concluded that the interventions had not been wholly successful in encouraging safer sexual behavior. The authors of both trials discuss the 
Cooper et al.

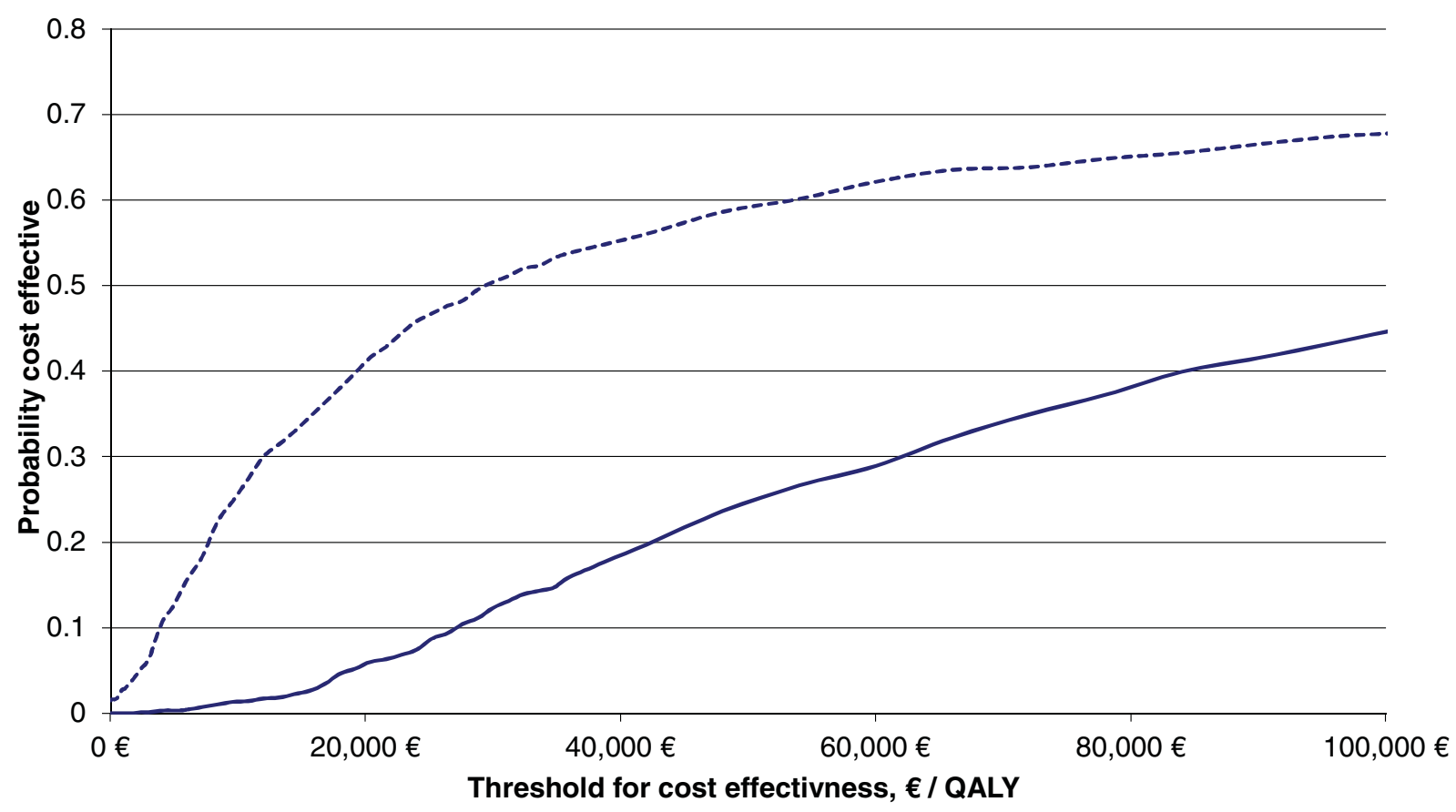

-Peer led ----Teacher led

Figure 1. Cost-effectiveness acceptability curves of the PSA results for 1,000 iterations for the teacher and peer led interventions versus standard care. QALY, quality-adjusted life-year.

likely reasons for this. Wight et al. concluded that, for some young people, the skills would not be put into practice until they had begun a sexual relationship, by which time the skills would likely be harder to recall (30). The results from our model also suggest that interventions would be better value for money if conducted at a later age.

The cost-effectiveness of school-based interventions has previously been evaluated for "Safer Choices," a 2-year, theorybased, multi-component HIV/STI prevention intervention in the United States $(6 ; 26)$. The conclusions from these evaluations varied widely: one study (6) found the intervention was not cost-effective with a cost per STI case averted $>\$ 39$ million. In contrast, the other (26) found the same intervention was cost saving. The difference in the results between these two studies may be partly explained by the fact that Wang et al. (26) included the effects of the intervention on other STIs and unintended pregnancy; while these effects were not included by Cohen et al. (6)

The current study incorporated the impact of changes in HRQoL to individuals from the STIs to present the results in terms of cost per QALYs gained, which are commonly used by health decision makers (18). Few studies that have estimated the cost-effectiveness of interventions to prevent STIs have used estimates of QALYs (2). Rather, they have used other outcome measures such as cost per major outcome averted or cost per case avoided (16;26), which makes them more difficult to compare with other health interventions, and involves making assumptions such as assuming that all STIs have equal health consequences, which is unlikely to be the case.
The evaluation of public health and health promotion interventions presents particular methodological challenges compared with the evaluation of clinical interventions (27). Decision makers, such as NICE, prefer evidence comparing the relevant alternatives to come from randomized controlled trials (RCTs) where available. However, there are fewer controlled trials of public health interventions, and these tend to be heterogeneous, of variable methodological quality and with short follow-up. Economic evaluation requires estimation of long-term outcomes, with health outcomes typically measured in QALYs, which are often difficult to quantify within a public health context. Public health interventions are often wide ranging with the cost and benefits associated with an intervention falling on many parts of the public sector (e.g. education and health services). Finally, the data used to build economic models are often scarce and several assumptions may have to be made.

Our economic evaluation is one of the few published examples of an assessment of the cost-effectiveness of school-based behavioral interventions, particularly in the United Kingdom. It was informed by a systematic review of RCTs of the effectiveness of the interventions, systematic review of previous cost-effectiveness studies, and systematic searches for the input parameter data. Despite these strengths, it is subject to the following limitations. Although the results from the systematic review of effectiveness showed that these programs can bring about improvements in knowledge, and increased self-efficacy, the meta-analysis did not show a statistically significant intervention effect for behavioral outcomes. However from a health 
economic framework, Bayesian statistical techniques such as probabilistic sensitivity analysis are useful to describe the uncertainty around the results. In this case, we have used the best available data and shown the probability that the intervention will be beneficial and / or cost-effective compared with standard sex education.

Furthermore, although teacher-training behavioral interventions and peer-led behavioral interventions are both compared with standard sexual health education, there is no direct evidence comparing peer-led and teacher-led behavioral interventions. The differences in the results are due to the difference in the costs of the two interventions, whereby the teacher-led behavioral intervention was cheaper due to the need for less frequent training. We have assumed the same effect from both interventions (as the intervention effect has been derived from a meta-analysis for behavioral interventions versus standard sex education).

For many of the parameters for the economic evaluation, there were no available data for the $<16$-year-old age group and we have had to make assumptions to extrapolate data from older teenagers group. In particular, data on the sexual behavior of under-16s is needed. Often data are presented for the 18- to 25 -year-old age group in national surveys, and we recommend that the age groups used are extended.

\section{CONCLUSION}

School-based behavioral interventions which provide information and teach young people sexual health skills can bring about improvements in knowledge and increased self-efficacy, although these may be limited in terms of impact on sexual behavior. These interventions have been evaluated using an economic model that describes the cost and benefits associated with reductions in STIs due to improved sexual behavior. There is uncertainty around the results of our economic evaluation due to the uncertainty around the effect of the intervention on sexual behavioral outcomes. The model results were most sensitive to changes in parameter values for the intervention effect and the transmission probability of STIs. Teacher-led interventions are likely to be cheaper than peer-led interventions due to less frequent retraining. Further economic evaluation, integrated into primary evaluation, is needed.

\section{CONTACT INFORMATION}

Dr Keith Cooper, Southampton Health Technology Assessments Centre (SHTAC), 1st Floor, Epsilon House, Enterprise Road, Southampton Science Park, University of Southampton, Southampton, SO16 7NS, UK. Tel 02380595631

Dr Jonathan Shepherd, SHTAC, University of Southampton, Southampton, SO16 7NS, UK

Dr Joanne Picot, SHTAC, University of Southampton, Southampton, SO16 7NS, UK
Dr Jeremy Jones, SHTAC University of Southampton, Southampton, SO16 7NS, UK

Josephine Kavanagh, The EPPI-Centre, University of London Prof Angela Harden, Institute for Health and Human Development, University of East London, London

Elaine Barnett-Page, The EPPI-Centre, University of London Prof Andrew Clegg, SHTAC, University of Southampton, Southampton, SO16 7NS, UK

Dr Debbie Hartwell, SHTAC, University of Southampton, Southampton, SO16 7NS, UK

Dr Geoff Frampton, SHTAC, University of Southampton, Southampton, SO16 7NS, UK

Alison Price, Wessex Institute, University of Southampton, Southampton, SO16 7NS, UK

\section{CONFLITS OF INTEREST}

Keith Cooper, Jonathan Shepherd, Jo Picot, Jeremy Jones, Angela Harden, Elaine Barnett-Page, Andy Clegg, Debbie Hartwell and Alison Price have received a grant to their institution from NIHR Health Technology Assessment Programme, Reference number 06/72/02. None of the authors have any conflicts of interest.

\section{REFERENCES}

1. Adams EJ, Turner KM, Edmunds WJ. The cost effectiveness of opportunistic chlamydia screening in England. Sex Transm Infect. 2007;83:267274

2. Barham L, Lewis D, Latimer N. One to one interventions to reduce sexually transmitted infections and under the age of 18 conceptions: A systematic review of the economic evaluations. Sex Transm Infect. 2007;83:441-446.

3. Brown RE, Breugelmans JG, Theodoratou D, Benard S. Costs of detection and treatment of cervical cancer, cervical dysplasia and genital warts in the UK. Curr Med Res Opin. 2006;22:663-670.

4. Chesson HW, Ekwueme DU, Saraiya M, Markowitz LE. Costeffectiveness of human papillomavirus vaccination in the United States. Emerg Infect Dis. 2008;14:244-251.

5. Chlamydia Advisory Group. The first steps... Annual report of the National Chlamydia Screening Programme in England. London: Department of Health; 2004:1-44.

6. Cohen DA, Wu SY, Farley TA. Comparing the cost-effectiveness of HIV prevention interventions (DARE structured abstract). $J$ Acquir Immune Defic Syndr. 2004;37:1404-1414.

7. Curtis L, Netten A. Unit costs of health and social care. Canterbury, PSSRU, University of Kent; 2006.

8. Fisher M, Benn P, Evans B, et al. UK Guideline for the use of postexposure prophylaxis for HIV following sexual exposure. Int J STD AIDS. 2006;17:81-92.

9. Health Protection Agency. Diagnoses and rates of selected STIs seen at UK GUM clinics by country and age group: 2002-2006. London: Health Protection Agency, Centre for Infections; 2007:1-33.

10. Health Protection Agency. Testing times HIV and other sexually transmitted infections in the United Kingdom. London: Health Protection Agency, Centre for Infections; 2007.

11. Howell-Jones R. Baseline HPV epidemiology studies. http://www. cornwall.nhs.uk/CornishMicrobiologicalSociety/Presentations/PDF/ HPVEpidemiologyRHowellJones.pdf 2008 (accessed October 1, 2008). 
12. Hu D, Hook EW III, Goldie SJ. Screening for Chlamydia trachomatis in women 15 to 29 years of age: A cost-effectiveness analysis. Ann Intern Med. 2004;141:501-513.

13. Johnson AM, Mercer CH, Erens B, et al. Sexual behaviour in Britain: Partnerships, practices, and HIV risk behaviours. Lancet. 2001;358:18351842.

14. Lader D. Contraception and sexual health. Omnibus Survey Report No. 33. -108. Newport: Office of National Statistics; 2007.

15. Langley PC, White DJ, Drake SM. The costs of treating external genital warts in England and Wales: A treatment pattern analysis. Int J STD AIDS. 2004;15:501-508.

16. Low N, McCarthy A, Macleod J, et al. Epidemiological, social, diagnostic and economic evaluation of population screening for genital chlamydial infection. Health Technol Assess. 2007;11:iii-xii, 1.

17. Miners AH, Sabin CA, Trueman P, et al. Assessing the cost-effectiveness of HAART for adults with HIV in England. HIV Med. 2001;2:5258.

18. NICE. Guide to the methods of technology appraisal. London: National Institute for Health and Clinical Excellence; 2008.

19. Quinn TC, Gaydos C, Shepherd M, et al. Epidemiologic and microbiologic correlates of Chlamydia trachomatis infection in sexual partnerships. JAMA. 1996;276:1737-1742.

20. Rao GG, Bacon L, Evans J, et al. Prevalence of Neisseria gonorrhoeae infection in young subjects attending community clinics in South London. Sex Transm Infect. 2008;84:117-121.

21. Rothenberg R, Potterat JJ, Koplan JP. The algebra of condoms and abstinence. Sex Transm Dis. 2005;32:252-254.

22. Shepherd J, Kavanagh J, Picot J, et al. The effectiveness and costeffectiveness of behavioural interventions for the prevention of sexually transmitted infections in young people aged 13-19: A system- atic review and economic evaluation. Health Technol Assess. 2010;14: 1-iv.

23. Smith KJ, Tsevat J, Ness RB, Wiesenfeld HC, Roberts MS. Quality of life utilities for pelvic inflammatory disease health States. Sex Transm Dis. 2008;35:307-311.

24. Stephenson JM, Strange V, Forrest S, et al. Pupil-led sex education in England (RIPPLE study): Cluster-randomised intervention trial. Lancet. 2004;364:338-346.

25. Tengs TO, Lin TH. A meta-analysis of utility estimates for HIV/AIDS. [see comment]. Med Decis Making. 2002;22:475-481.

26. Wang LY, Davis M, Robin L, et al. Economic evaluation of safer choices: A school-based human immunodeficiency virus, other sexually transmitted diseases, and pregnancy prevention program. Arch Pediatr Adolesc Med. 2000;154:1017-1024.

27. Weatherly H, Drummond M, Claxton K, et al. Methods for assessing the cost-effectiveness of public health interventions: Key challenges and recommendations. Health Policy. 2009;93:85-92.

28. Weinstein M, Graham J, Siegel J. Cost effectiveness analysis of AIDS prevention programs: Concepts, complications, and illustrations. In: Turner C, Miller H, Moses L, eds. AIDS: Sexual behavior and intravenous drug use. Washington, DC: National Academy Press; 1989:471-499.

29. WHO. In: Currie C, Gabhainn S, Godeau E, Roberts C, Smith R, eds. Inequalities in young people's health. HBSC international report from the 2005/2006 survey. Copenhagen, Denmark: WHO; 2008:1-210.

30. Wight D, Raab GM, Henderson M, et al. Limits of teacher delivered sex education: Interim behavioural outcomes from randomised trial. BMJ. 2002;324:1430.

31. Wolstenholme JL, Whynes DK. Stage-specific treatment costs for cervical cancer in the United Kingdom. Eur J Cancer. 1998;34:1889-1893. 